\title{
Linx
}

Revue des linguistes de l'université Paris X Nanterre

$76 \mid 2018$

Dire l'humain : les noms généraux dénotant les

humains

\section{Étude contrastive anglais-français de noms d'humains dans un corpus contraint}

Maryvonne Boisseau

\section{(2) OpenEdition}

Journals

Édition électronique

URL : http://journals.openedition.org/linx/2469

DOI : $10.4000 /$ linx.2469

ISSN : 2118-9692

Éditeur

Presses universitaires de Paris Nanterre

Édition imprimée

Date de publication : 31 juillet 2018

Pagination : 163-183

ISSN : 0246-8743

Référence électronique

Maryvonne Boisseau, «Étude contrastive anglais-français de noms d'humains dans un corpus contraint », Linx [En ligne], 76 | 2018, mis en ligne le 31 janvier 2019, consulté le 30 avril 2019. URL: http://journals.openedition.org/linx/2469; DOI : 10.4000/linx.2469

Ce document a été généré automatiquement le 30 avril 2019

Département de Sciences du langage, Université Paris Ouest 


\title{
Étude contrastive anglais-français de noms d'humains dans un corpus contraint
}

\author{
Maryvonne Boisseau
}

\section{Introduction}

1 C'est généralement par le biais de classifications que les phénomènes linguistiques touchant aux valeurs sémantiques des catégories du discours sont abordés. C'est ainsi le cas pour une sous-catégorie de noms désignés de «noms d'humains » pour lesquels une recherche visant à dégager leurs caractéristiques au travers de leurs emplois est en cours ${ }^{1}$ . En réalité leur présence dans les textes de tous ordres, oraux et écrits, est si fréquente qu'elle passe souvent pour évidente et reste inaperçue. Or le nom d'humain dit nécessairement quelque chose d'un rapport au monde et des relations que les humains entretiennent avec leur environnement social et, à cet égard, cette sous-catégorie n'a pas encore reçu toute l'attention qu'elle mérite. Les études conduites à ce jour, principalement pour le français ${ }^{2}$, s'intéressent à l'usage dit ordinaire dans des contextes généralement monolingues (français, allemand, espagnol, portugais), à l'aide de grands corpus électroniques. Cet article se propose d'aborder la question d'un autre point de vue, celui de la traduction. L'observation conjointe d'un texte original et de sa traduction en une autre langue (ou en d'autres langues) permet en effet de mieux mettre en évidence, à travers les différences entre les langues, le fonctionnement même des langues en présence dont la spécificité s'apprécie alors au regard des récurrences éventuelles dans la manière de traduire ou dans les écarts contraints par la grammaire et les usages propres à chacune d'elles.

2 Le corpus retenu est composé d'un ensemble de poèmes en langue anglaise du poète irlandais Derek Mahon traduits en français par Jacques Chuto. L'œuvre poétique de Mahon présente un intérêt particulier pour cette exploration de la sémantique des noms d'humains du fait d'une asymétrie patente entre les occurrences de noms d'humains et 
celles de noms d'objets qui pullulent dans l'ensemble de l'œuvre. Cependant, notre propos ne cherche pas à étayer dans l'immédiat une hypothèse herméneutique mais, dans une perspective strictement linguistique et contrastive, à dégager à partir de la comparaison interlinguistique, les valeurs sémantiques des noms sélectionnés qui justifient les choix de traduction.

3 Aussi après avoir rappelé ce que les grammaires anglaises et françaises disent de cette catégorie non identifiée en tant que telle, nous aborderons la question du corpus, sa nature et ses limites, pour examiner ensuite de manière empirique les occurrences les mieux représentées, à savoir man et people, dans un va-et-vient entre les deux langues. Nous nous appuierons sur les travaux du groupe NHUMA espérant contribuer, par cette étude, à la reconnaissance d'une sous-catégorie de noms encore indifférenciée au sein de la classe des noms.

\section{Le nom d'humain dans les grammaires : un aperçu}

\subsection{Grammaires anglaises}

4 Pas plus en anglais qu'en français la sous-catégorie spécifique des noms d'humains n'a véritablement retenu l'attention des grammairiens et des linguistes qui, de manière générale, rangent ce qu'on appelle ici «nom d'humain » sous l'étiquette " animé » et/ou "collectif», deux des traits sémantiques sur lesquels s'établissent certaines classifications des noms. On retrouve dans les grammaires anglaises une manière de catégoriser les noms très proche de celles des grammaires françaises, fondée sur des traits à la fois sémantiques et syntaxiques. Ainsi Quirk \& al. (1972: 127-135) organisent-ils l'étude de la typologie des noms de la façon suivante (127):

Proper/common, count/mass

Nouns with dual membership

Countability

Gradability

Words in -ing : a survey,

tandis que Huddleston \& Pullum (2012 [2002] : 328) divisent la catégorie des groupes nominaux en trois sous-classes principales : les noms communs, les noms propres et les pronoms. Ils traitent ensuite de l'interprétation des noms en dénombrables et indénombrables (count and non count nouns, id. : 334), soulignant le caractère sémantique $\mathrm{du}$ critère: «It follows that when we speak of count nouns and non count nouns it is to be understood that we are concerned with nouns as used with a count and non count interpretation respectively" (id.: 335, notre soulignement). Une autre grammaire (Depraetere \& Langford, 2012), plus récente que les deux grammaires de référence citées, reprend cependant d'autres termes courants en matière de typologie nominale dans son paragraphe «Types of nouns»:

Noun scan be classified on the basis of a number of criteria. A noun may denote something concrete (such as rain, table or factory) or something abstract (such as happiness, bravery or trust). From a usage point of view, the concrete $\sim$ abstract distinction does not play a vital role, in the sense that it has no impact on the choice of the other forms. [...]

Another distinction concerns the difference between common nouns (such as dog, cat, woman) and proper nouns (such as Rover, Fluffy, or Rebecca). As we will see below, this distinction can sometimes be relevant when it comes to the use of the article [...]. 
The distinction between animate nouns (such as girl or shopkeeper) and inanimate nouns (such as coffee table or television set) determines the choice of Subject or object pronouns in the third person. [...] The animate inanimate inanimate distinction is important to speakers whose native language has nouns obligatorily falling into different grammatical genders [...]. One final distinction [...] concerns individuating nouns and collective nouns [...].

The above distinctions are all secondary to a major distinction that is crucial for the choice and use of determiners in English as well as for Subject-verb agreement: the distinction between countable and uncountable nouns (2012:79-80, soulignement dans le texte).

La situation est en fait résumée dans la grammaire, plus ancienne, de Sylvia Chalker (1987 [1984]: 25):

Traditional grammar recognised that there were nouns of different kinds, and defined them - as it did nouns as a whole - on notional grounds. A typical classification was into common nouns (subdivided into abstract and concrete), proper nouns (ie names) and collective nouns. But the abstract-concrete distinction can be difficult to apply (Which is laughter or a laugh?) and the classification as a whole is not a reliable guide to grammatical usage. Modern grammar therefore makes formal distinctions. Two of the labels (proper and collective) are retained, but redefined into strictly formal terms while the major division is into count and mass.

7 Il n'est ainsi fait nulle part mention, dans ces ouvrages consultés, d'une sous-catégorie spécifique correspondant à « noms d'humains ». Trois des dichotomies relevées dans ces présentations, propre/commun, singularisant/collectif et dénombrable/indénombrable, peuvent en effet leur être appliquées, mais la distinction concret/abstrait et l'opposition animé/non animé n'ont guère de sens les concernant puisque, a priori, les traits +concret ou +animé constituent des caractéristiques qui sont propres aux entités qu'ils représentent, au sens où ce à quoi ils réfèrent est aisément identifiable pour des raisons principalement ontologiques et extra-linguistiques. On notera enfin que les oppositions relèvent à la fois du «notionnel ", généralement perçu comme ce qui relève du sémantique, et du discursif-syntaxique puisque l'opposition count et mass, conditionne le fonctionnement de ces entités en discours selon la compatibilité avec tel ou tel déterminant et l'accord en genre et en nombre, selon les langues.

\subsection{Grammaires françaises}

8 Pour ce qui est du français, la situation est résumée par Schnedecker 2015 (in S \& M, 2015) dans un article qui s'interroge sur la spécificité de cette catégorie relativement négligée par les linguistes alors qu'elle « est bien établie dans les grammaires tant du point de vue terminologique que définitoire » $(2015: 5)$. Selon Schnedecker (2015:10-17), les linguistes qui se sont intéressés à cette catégorie l'ont fait incidemment pour des raisons externes (comme, par exemple, la féminisation des noms de métier), ou pour des raisons internes (morphologie, syntaxe, sémantique). La conséquence en est une difficulté de définition de la catégorie elle-même. À partir de la classification de Gross (1995), « une incontestable avancée » (id.: 18), l'article explique alors les difficultés de catégorisation qui existent pour cette sous-classe de noms. Il passe en revue les problèmes, questions et différents arguments à prendre en compte lors de l'établissement des critères de classement d'ordre divers et met ainsi en évidence la diversité d'une classe de noms qui ne se laisse pas appréhender aussi facilement que l'étiquette « nom d'humain » pourrait le laisser croire. 


\section{Le corpus}

\subsection{La Mer hivernale \& autres poèmes}

Notre corpus est constitué d'un recueil de quarante-quatre poèmes incluant deux séquences de trois poèmes chacune, soit quarante-huit textes accompagnés de leur traduction, ce qui fait un total de quatre-vingt-seize textes. Intitulé La Mer hivernale \& autres poèmes, cet ensemble de textes choisis par le traducteur, Jacques Chuto, ne représente qu'environ 22\% de l'œuvre poétique de Derek Mahon publiée à la date de 2011 et rassemblée dans un volume intitulé New Collected Poems (2011) (désormais NCP). Les deux-cent quatorze poèmes publiés dans $N C P$, auxquels il faut ajouter d'autres volumes de traductions et de textes critiques en prose, couvrent une carrière poétique et littéraire de quelque quarante années. Internationalement reconnu comme un immense poète (et un grand traducteur), Mahon se distingue par une écriture musicale, une langue très travaillée, d'une grande richesse syntaxique et lexicale - et cela seul suffirait à justifier le choix de ce poète-làa ${ }^{3}$, témoignant d'un rapport esthétique au monde dominé par une vision ironique d'une réalité envahie par des choses et des objets, artefacts de tous ordres, depuis l'objet d'art jusqu'à l'objet utilitaire abandonné, mis au rebut, déchet permanent de notre civilisation. Cette présence massive des objets et, par conséquent, d'un point de vue linguistique, des noms communs qui les désignent, amène alors à s'interroger sur la présence de l'humain dans une poésie peuplée d'objets auxquels elle semble donner vie, ou à tout le moins sur la façon dont est poétisé, à travers la langue, le rapport de l'homme à l'objet et à la réalité qui l'entoure. La présence de l'humain, perçue comme non saillante, peut se manifester de diverses façons, mais la manière la plus évidente est d'abord la désignation et la dénomination des humains eux-mêmes, au moyen de «noms d'humain », objets de cette étude ${ }^{4}$.

\subsection{Un corpus contraint}

Le choix d'un "corpus contraint ", alors que la linguistique se déploie maintenant dans les grands corpus électroniques, les données quantitatives et les statistiques, n'est pas anodin. S'il est désormais indéniable que l'exploitation de ces grands corpus permet de renouveler la description de faits linguistiques, un corpus contraint présente aussi des avantages, en dépit des difficultés méthodologiques et techniques que son exploration peut poser ${ }^{5}$. Par " corpus contraint ${ }^{6}$ ", nous entendons, à la suite de Culioli, un ensemble de textes, au sens restreint de textes écrits dont on ne peut défaire la cohérence interne : «[...] le texte suivi à support écrit possède ses propres contraintes linguistiques: [...] règles de production et de reconnaissance [...], règles de cohérence [...] et de modes de construction des valeurs référentielles" (Culioli, 1984: 9). Dans le contexte de cette étude, ce sont les "valeurs référentielles» de certains des noms d'humains répertoriés dans le corpus, portées par le cotexte étroit d'un poème, le contexte élargi du recueil ainsi que par le contexte translinguistique construit par la traduction, qui seront l'objet de nos descriptions et analyses. Notre approche des noms d'humains, indirecte et contrastive, est ainsi motivée par la nature des textes soumis à l'étude (voir 2.1. supra) et, par une hypothèse interprétative qui, inversant la problématique de l'omniprésence, sous de multiples formes, de l'objet dans cette poésie, vise à mettre en lumière son corollaire, une discrète présence de l'humain, dont les diverses mentions, dans cet ensemble de textes, 
font apparaitre non seulement les relations internes, mais également les modalités d'un rapport au monde subordonnant in fine les objets à un point de vue et à un regard humains.

\subsection{Approche quantitative du corpus et observations préliminaires}

11 Sur les quarante-huit textes du corpus, trente-huit contiennent des noms d'humains, soit environ $80 \%$ des textes, le nombre d'occurrences dans un texte variant suivant la longueur du poème. Le nombre d'exemples relevés s'élève à 89 et le nombre d'occurrences à 145 (un exemple peut compter plusieurs occurrences), auquel il faut ajouter cinq occurrences présentes dans les titres, portant ainsi le nombre d'occurrences à 150. Ces cinq titres, An Unborn Child, No Rest for the Wicked, Ecclesiastes, The Last of the Fire Kings, Girls on the Bridge, donnent un aperçu de trois sous-catégories de noms d'humains représentés : noms de phase, noms de groupe, noms de fonction. Les souscatégories les mieux représentées sont celles:

12 - des noms généraux avec man et mankind (respectivement neuf et une occurrences) et people (six occurrences), les noms de phase avec child/children (sept occurrences), girl (six occurrences) et boy (deux occurrences),

13 - des noms de relation avec friend-s (six occurrences), mother (quatre occurrences), wife/ wives (quatre occurrences), husband (une occurrence), daughter (deux occurrences) et son (une seule occurrence).

14 Les noms de métiers (ex. anthropologist) et de fonction (ex. king) ou, plus largement, selon la terminologie de De Swart et al. (20077) des noms de capacité sont représentés à travers diverses occurrences uniques à l'exception de poet (trois occurrences), tout comme le sont des noms dits « de qualité » ou d'« insulte » (par exemple idiot) (voir Schnedecker 2015 : 25), ou bien encore des noms collectifs (groupes et entités) et des noms distinguant un état, qu'il soit épisodique ou permanent (ex. : fools and mad, saints and scholars).

15 À ce stade, notons que certaines sous-catégories sont ainsi mieux représentées que d'autres: la très grande majorité des occurrences appartient, en effet, à la catégorie centrale des noms d'humains. On relève néanmoins quelques unités lexicales dénotant des « événements » sous-jacents (noms épisodiques comme tourists, visitors) et des items périphériques (the clamorous dead). Par ailleurs, si tous les noms d'humains sont traduits, de rares cas de glissements métonymiques font apparaître une traduction par un nom d'humain là où il n'y en pas dans l'original et inversement.

\section{Description et analyses}

\subsection{Choix des exemples}

16 Idéalement, chaque énoncé dans lequel se trouve au moins une occurrence de nom d'humain devrait être examiné et comparé à sa traduction. Le format de cette étude ne permet pas cette comparaison exhaustive, ce qui amène à n'engager qu'une exploration partielle du corpus sur la base d'une sélection d'énoncés. Cette sélection répond à trois critères : i) l'occurrence du nom d'humain appartient à la sous-catégorie manifestement la mieux représentée, les "noms généraux ", ii) les diverses occurrences permettent de vérifier les traits et valeurs référentielles que les choix de traduction mettent en lumière, 
iii) la nature plus marginale de certaines autres occurrences peut apporter un éclairage différent.

Nous nous aiderons donc des classifications existantes, celle de Gross citée dans Schnedecker $(2015: 18)$ et celle issue de la recherche de Gosselin qui procède à une classification des noms d'humains selon le type de modalité, intrinsèque et/ou extrinsèque, que le nom exprime.

\subsection{Série $1: \operatorname{man}$}

L'entrée man dans les dictionnaires, comme on peut s'y attendre, montre la diversité de ses emplois. Quels que soient les dictionnaires, la première définition offerte correspond à son emploi de nom dénombrable (countable) et, du point de vue de sa valeur sémantique, à l'opposition des genres (selon le sexe) : "an adult human male ${ }^{8}$ ", «male person 1 [C] an adult male human : a good-looking young $\operatorname{man}^{9}$ ", « $1[C]$ an adult human male ${ }^{10}$ ", «S: (n) man, adult male - (an adult person who is male (as opposed to a woman); " there were two women and six men on the bus ") ${ }^{11}$ ». Son emploi en tant que nom indénombrable ne vient qu'en second lieu, voire bien après dans certaines de ces entrées, qui listent également les variations de registre susceptibles d'affecter sa saisie. Une explication diachronique et sociolinguistique intéressante est fournie à ce propos par le NODE $(1998: 1122)$ :

USAGE Traditionally the word man has been used to refer not only to adult males but also to human beings in general, regardless of sex. There is a historical explanation for this: in Old English the principal sense of man was 'a human being', and the word wer and wif were used to refer specifically to 'a male person' and 'a female person' respectively. Subsequently man replaced wer as the normal term for 'a male person', but at the same time the older sense 'a human being' remained in use.

In the second half of the twentieth century the generic use of man to refer to 'human beings' in general (as in reptiles were here long before man appeared on the earth) became problematic; the use is now often regarded as sexist or at best old-fashioned. In some contexts, alternative terms such as the human race or humankind may be used. Fixed phrases and sayings such as time and tide wait for no man can be easily rephrased, e.g. time and tide wait for nobody. However, in other cases particularly in compound forms, alternatives have not yet become established: there are no standard accepted alternatives for manpower or the verb man, for instance.

19 Cet encadré est intéressant en ce qu'il attire l'attention sur l'évolution de l'usage du nom sous la pression des changements sociétaux, la perception - en termes de registre - que l'on peut avoir de la persistance d'un usage ancien, la concurrence des deux valeurs, individualisante et générique, toujours présentes dans l'usage aujourd'hui et, incidemment, la disparition de wer alors que s'est maintenu wif devenu wife et renvoyant aujourd'hui à épouse, ainsi que la tendance à utiliser le pronom comme substitut neutre à valeur indéfinie, indéterminée et générique, en lieu et place du terme le plus précis.

En français, l'entrée homme dans le Grand Robert de la Langue Française (2001) occupe à elle seule vingt et une colonnes, soit dix pages (1856-1866). Contrairement aux entrées des dictionnaires anglais, c'est la valeur notionnelle qui est répertoriée en premier tandis que la valeur individualisante, incluse dans la notion, vient en second :

I Être appartenant à l'espèce animale la plus évoluée de la Terre. $\rightarrow$-anthrope, anthropo-. L'homme, les hommes. $\rightarrow$ Humanité ; gens. Un homme. $\rightarrow$ Individu, personne. - REM. En ce sens homme désigne l'humain mâle ( $\rightarrow$ ci-dessous, homme 
au sens II) ou femelle ( $\rightarrow$ Femme); il ne peut s'appliquer qu'à des mâles (hommes au sens II), mais jamais à des femmes exclusivement. Le caractère abstrait de cet emploi, où les hommes correspond à les humains, explique la fréquence au singulier collectif l'homme («l'humanité »). Dans ce dernier cas, le pluriel peut désigner une pluralité de classes (les hommes fossiles) et non une pluralité d'individus. Enfin, l'emploi de l'homme (individuel) dans ce sens est rendu difficile par l'ambiguïté avec le sens II. Pour éviter ces ambiguïtés, possibles aussi avec le pluriel, on emploi parfois un humain, les humains.

[...]

II (Fin $X^{e}$, Passion du Christ). Être humain mâle, et (le plus souvent) adulte. $\rightarrow$ Garçon (cit. 11), mâle, masculin ; -andre, -andrie (2.), andro-, vir-. - REM. Dans ce sens plus concret, c'est l'emploi individuel (un, des hommes) qui l'emporte sur le général (l'homme) ; spécialt. « mâle adulte de l'espèce humaine ${ }^{12}$.

21 Les extraits ci-dessous illustrent ces acceptions répertoriées et ne montrent pas d'emploi surprenant de ce nom d'humain général. Les choix de traduction mettent cependant en relief une valeur référentielle construite par le contexte plus précise que l'acception générale.

(1) 'Wonders are many and none is more wonderful than man' $\left(2-22^{13}\right)$

(1a) «Les prodiges sont légion, mais aucun n'est plus étonnant que l'homme » (2a-23)

Dans ce vers (1), qui est une traduction de paroles du chœur dans l'Antigone de Sophocle et se trouve être le premier vers d'un poème dans lequel Mahon ne se montre guère bienveillant à l'égard de l'espèce humaine, le nom man renvoie justement de façon générale à l'espèce humaine elle-même, sans filtre puisque l'absence de déterminant en anglais, marque ce qu'on désigne, en termes culioliens, de "renvoi à la notion ». La traduction par l'article le marque la même opération abstraite totalement généralisante. Les deux langues sont ici très proches dans leur fonctionnement à cette différence près qu'il n'y a pas de superposition possible des déterminants : le déterminant the [the man] n'aurait pas renvoyé au degré d'abstraction de man recherché ici (c'est-à-dire le plus haut degré d'abstraction possible). Il est par ailleurs très peu probable que cette occurrence, en anglais comme en français, soit perçue, dans ce contexte poétique, comme relevant d'un emploi "sexiste» ou «vieux jeu», ce qui semblerait indiquer l'influence du genre (poétique) sur la construction même des valeurs de l'occurrence.

(2) [...] but the wise man knows / To cleave to the one living absolute / Beyond paraphrase $[. .].(10-26)$

(2a) [...] mais le sage sait / S'attacher à l'unique et vivace absolu / Qui passe la paraphrase, [...] (10a-27)

(3) [...]. The showers of / April, flowers of May mean nothing to me, nor the / Late light of June, when my gardener / Describes to strangers how the old man stays in bed / On seaward mornings after nights of / Wind, takes his morphine and will see no one. [...] (13-34)

(3a) [...]. Les averses / D'avril, les fleurs de mai me laissent froid, tout comme / La lumière des soirs de juin où mon jardinier / Explique aux curieux que le vieux reste au lit / Quand la terre dérive vers la mer après les nuits / De vent, qu'il prend sa cocaïne, et ne veut voir personne. (13a-35)

(4) And a warm briar gurgled / When the old man talked to himself; // But the doorbell seldom rang / After the milkman went, (38-66)

(4a) Et une pipe chaude gargouillait / Quand le vieil homme se parlait à lui-même;

// Mais on sonnait rarement, / Une fois le laitier passé (38a-67)

23 Les occurrences de man dans les extraits $(2 / 2 a),(3 / 3 a)$ et $(4 / 4 a)$ sont qualifiées par un adjectif (wise et old) et sont, formellement, exactement parallèles. Or, l'une, sans être aussi 
abstraite que $\varnothing$ man, renvoie à un sous-groupe de man représenté par un individu exemplaire (prototypique) tandis que les deuxième et troisième renvoient à un individu particulier.

Dans (2/2a), la valeur générique de l'occurrence de the wise man, confirmée par le présent simple du verbe know, est indissociable de la présence de l'adjectif qui introduit une appréciation positive proche d'une modalité appréciative dans une expression que l'on peut assimiler à une collocation (wise man, The Three Wise Men). L'indissociabilité de l'adjectif et du nom aboutit, de fait, à la mise en valeur de la qualification - comme si la tête du syntagme (le nom) n'était qu'un support pour le sens porté par l'élément qualifiant - et le groupe nominal est alors traduit par le sage, occurrence de nom d'humain par conversion de l'adjectif, porteuse, si l'on suit Gosselin ${ }^{14}$, d'une modalité aléthique (classifiante) et positive. Les extraits $(3 / 3 a)$ et $(4 / 4 a)$, en revanche, renvoient à un individu, l'énonciateur-locuteur ( $m e$ ) dans le premier cas, dans une instance de discours rapporté où ce I devient the old man; et, dans l'extrait (4/4a), il s'agit de celui dont on parle, le thème, au sens métalinguistique, et le sujet du poème. L'adjectif ne semble ici n'avoir qu'une valeur descriptive et pourrait relever d'une valeur épistémique évaluative. Cependant, l'expression est, tout comme la précédente, relativement fixée et appartient à un registre familier. La traduction retient là encore la qualification pour désigner la personne à laquelle l'occurrence renvoie dans le premier cas, le vieux, avec une connotation péjorative, alors que dans le second, la traduction par le vieil homme attire l'attention sur une connotation affective positive.

Ce qui semble se faire jour ici, c'est que la modalité du nom d'humain général, si modalité il y a véritablement, lui est conférée par l'adjectif qui l'accompagne (ce serait donc une modalité extrinsèque). La valeur de l'adjectif est transférée sur le nom au moyen de ce que, dans le cadre de la Théorie des Opérations Prédicatives et Énonciatives élaborée par Culioli (TOPE), on appellerait une opération de filtrage faisant intervenir la subjectivité d'un énonciateur. Enfin, l'adjectif seul ne suffit pas puisque dans une structure strictement parallèle, la valeur qualitative ajoutée peut aussi bien tendre vers une valeur négative que vers une valeur positive.

(5) A hundred men imagine / Love when I drink wine, / and then I begin to think of your words and mine. (18-40)

(5a) Tant d'hommes rêvent d'amour / lorsque je bois du vin; / je me mets alors à penser / à tes mots et aux miens. (18a-41)

(6) Elsewhere they are burning / Witches and heretics / In the boiling squares, // Thousands have died since dawn / In the service / of barbarous kings; (30-54)

(6a) Ailleurs on brûle vifs / Hérétiques et sorcières / Sur des places en ébullition, // Des milliers d'hommes sont morts / Depuis l'aube au service / De rois barbares; (30a-55)

L'intérêt des extraits (5/5a) et (6/6a) réside dans le traitement de la quantification. Dans $(5 / 5 a)$, la traduction dégage du grand nombre la seule valeur quantitative approximative contenue, paradoxalement, dans la précision de A hundred. Dans (6/6a), une contrainte syntaxique amène le traducteur à introduire le nom hommes clarifiant ainsi la répartition syntaxique des groupes, tous groupes de victimes, dans l'espace sémantique des deux vers, thousands, sujet syntaxique du verbe have died, n'est pas l'anaphore stricte des noms compléments witches et heretics.

(7) I want to be / Like the man who descends / At two milk churns / [...] Or the man / Who drops at night / From a moving train (31-56)

(7a) Je veux être pareil / à l'homme qui descend / Devant deux bidons de lait / [...]

Ou à celui / Qui se laisse tomber de nuit / D'un train en marche (31a-57) 
(8) We style, / as best we may, our private destiny, / or so it seems to me // as I chew my thumb / and try to figure out / what brought me to my present state - / an 'educated man', a man of consequence, no bum / but one who has hardly grasped what life is about, / if anything. (74-132)

(8a) Nous dessinons / tant bien que mal notre destin personnel ; / c'est du moins ce que je me dis, // en mâchonnant mon pouce / et m'efforçant de comprendre / comment j'ai fait pour en arriver là, / moi qui suis quelqu'un d'instruit, de connu, pas un clodo, / mais n'ai guère saisi à quoi rime la vie, / si elle rime. (74a-133)

27 (7/7a) et (8/8a) montrent le poids du déterminant et de la qualification dans la compréhension de l'identification, visée ( $I$ want to be) et indirecte via la comparaison en $(7 / 7 a)$, établie et directe ( $I=$ an 'educated man' = a man of consequence) en $(8 / 8 \mathrm{a})$, opposant man doté de propriétés et qualités positives, socialement reconnues, comme en témoignent les expressions figées constituant des unités polylexicales (modalités extrinsèques), à un exemplaire d'homme intrinsèquement blâmable (bum/clodo). On retrouve ici la possibilité d'interpréter les occurrences des noms en leur appliquant d'un côté la propriété agentive, intrinsèque à man/homme (7/7a) et, de l'autre (8/8a), certaines des valeurs modales dégagées par Gosselin (valeurs appréciatives internes et externes) qui conduisent à des choix de traduction en conséquence. Ces choix suivent de très près l'original tout en signalant, là encore, que c'est le marqueur de détermination et la qualification plus que le nom lui-même qui orientent vers le choix d'un pronom anaphorique (celui) ${ }^{15}$ ou d'un pronom indéfini (quelqu'un) soulignant le caractère relativement indéterminé de l'expression.

(9) That girl with her back to us waits / For her man to come home for his tea / Will wait till the paint disintegrates / And ruined dikes admit the esurient sea; (49-82)

(9a) Vue de dos, cette jeune femme qui attend / Que son homme rentre à la maison pour dîner / Attendra jusqu'à ce que la peinture s'effrite / Et que les digues cèdent à la mer vorace. (49a-83)

(10) The something rotten in the state / infects the innocent; the spite / mankind has brought to this infernal / backwater destroys the soul; / it sneaks into the daily life, / sunders the husband from the wife. (58-96)

(10a) Le quelque chose de pourri dans l'État / infecte les innocents; la haine apportée / par les hommes jusqu'à cet infernal / trou perdu dévaste les âmes, / se glisse dans la vie quotidienne, / sépare le mari de l'épouse. (58a-96a)

Ces deux derniers extraits, pour ce qui est du nom man, correspondent à des emplois bien répertoriés, du moins en ce qui concerne le registre familier pour her man et le sens de totalité plurielle pour mankind incluant tous les hommes et toutes les femmes perçus collectivement, soit l'humanité tout entière.

Dans (7/7a) l'occurrence de her man est repérée par rapport à That girl et cette relation de repérage lie les deux occurrences de telle sorte qu'un équivalent de man ici, tel que her husband, ou bien her lover, aurait créé une hétérogénéité linguistique au sein même de la prédication (alors même que le référent "extra-linguistique » ne varie pas) et, du point de vue sémantique global une forme d'incohérence. Il semble qu'il faille qu'une même modalité, ici aléthique, affecte les deux noms mis en relation. Mais c'est la traduction de girl par jeune femme, et non jeune fille, qui révèle en fait que c'est le « thème » de l'énoncé qui oriente l'interprétation vers la description d'une situation domestique banale et une scène de la vie quotidienne figée.

Dans (10/10a), la traduction de l'abstraction mankind par les hommes, désignant sans remords sexiste la totalité des hommes et des femmes, renforce la relation d'agentivité qui existe entre le sujet et le prédicat (verbe d'action) et met en relief, plus concrètement, 
la responsabilité des hommes dans la haine qui ronge la société en question ${ }^{16}$. Aussi, sousjacent à la représentation abstraite que suggère le nom collectif mankind, le trait +agent, plus ou moins masqué par le trait +classifiant $($ kind $=$ espèce $)$ devient saillant.

\subsection{Série 2 : people}

31 L'entrée people dans les dictionnaires ${ }^{17}$ est beaucoup moins fournie que celle de man. Il s'agit d'un nom collectif pluriel qui désigne de façon indifférenciée des êtres humains, hommes, femmes et enfants (première acception) et l'ensemble des personnes qui peuplent une nation (troisième ou quatrième acception recensée selon les dictionnaires, sing. ou plur.). People, c'est tout le monde et tout un chacun, les gens en somme, les personnes, pouvant désigner aussi bien la populace que l'ensemble des citoyens d'un pays, les employés d'une personne (ses gens), comme ses proches, dans un emploi d'ailleurs signalé comme "daté » par le NODE (1375) : «- (one's people) [dated] a person's parents or relatives: my people live in Warwickshire ».

Or, si l'on regarde les extraits suivants (11/11a à 15/15a), ce n'est pas l'emploi de people comme «nom général » qui domine mais bien plutôt son emploi dans deux acceptions moins étendues, celles de «peuple» d'une part et celle de "proches» et de « communauté particulière » d'autre part.

(11) [...] this is your / country, close one eye and be king. / Your people await you, their heavy washing / flaps for you in the housing estates - / a credulous people. (17-36)

(11a) [...] c'est ici ton / pays, ne ferme qu'un œil et te voilà roi ! / Peuple crédule, tes sujets t'attendent; leur linge / claque lourdement en ton honneur / dans les lotissements. (17a-37)

(12) Where the frugivorous / Inheritors recline / In their rich fabrics / Far from the sea. // But the fire-loving / People, rightly perhaps, / Will not countenance this (35-58)

(12a) Où les héritiers / frugivores se prélassent / Dans leurs riches étoffes / Loin de la mer. // Mais les adorateurs / Du feu, non sans raison peut-être, / Me refusent ce rêve (35a-59)

Sans nécessairement saisir le sens de ces deux extraits ${ }^{18}$, il apparait clairement que le nom people ne peut s'appréhender que dans sa relation aux deux autres noms d'humains présents dans ces vers, king (11/11a) et inheritors (12/12a). Cette relation, du point de vue de la référence, est celle d'un souverain ou d'une classe dominante à ses sujets, perçus ici comme une collectivité délimitée par le nombre indéterminé de ses membres et son appartenance. À ces propriétés générales s'ajoutent les qualifications plus précises qui, qualitativement, renforcent la saisie d'un ensemble délimité, ce qui n'en fait donc pas, en français l'équivalent de gens mais bien de peuple au sens de «Ensemble d'êtres humains vivant en société, habitant un territoire défini et ayant en commun un certain nombre de coutumes, d'institutions ${ }^{19}$ ». La traduction des deux extraits (11/11a) joue sur la nature de cette relation puisqu'elle la clarifie ${ }^{20}$ et propose deux termes spécifiques privilégiant un seul des traits sémantiques de people/peuple.

(13) Lost people of Treblinka and Pompeii ! (43-72)

(13a) Disparus de Treblinka, Pompéi ! (43a-73)

C'est dans cet exemple une autre communauté que le groupe nominal $N$ of $N$ construit, celle des personnes disparues lors de catastrophes de l'histoire ou naturelles, celle des clamorous dead mentionnée dans un autre poème. Le vocatif marqué par le point d'exclamation est un vocatif d'empathie qui rassemble, de manière provocante, les morts 
de Treblinka et ceux de Pompéi. S'il contribue, avec l'adjectif lost, à construire une modalité axiologique de la notion /people/, cette dernière demeure, semble-t-il, externe aux valeurs invariantes du nom lui-même, ce qui peut expliquer sa mise en relief par le choix de Disparus.

(14) He is far / From his people (23-46)

(14a) Il est loin des siens (23a-47)

(15) Walking along my own this windy morning / In a tide of sunlight between shower and shower (1-20)

(15a) En ce matin venteux, je me promène parmi les miens / Dans un flot de soleil au milieu des averses (1a-21)

L'occurrence de people dans (14/14a), tout comme son substitut par le pronom dans (15/15a) correspond à l'emploi cité comme "daté» par le NOED (voir supra). La collectivité que people désigne est restreinte à la sphère des proches et intimes, à la communauté étroite et locale dont on fait partie, avec laquelle on partage un sentiment d'appartenance. Le déterminant " possessif » dans les deux cas, his et my est le marqueur de cette relation et ce qu'il signifie dote people d'une valeur sous-catégorisante, celle d'une classe fermée constituée d'un nombre limité d'individus repérés par rapport à une personne et une seule.

Les emplois de people dans les extraits ci-dessus renvoient donc tous à l'idée de communauté et de relations et non, de façon remarquable, à l'emploi de people comme collectif rassemblant une pluralité indifférenciée. La pluralité désignée ici est située, identifiable et reconnaissable. Cet emploi (dont il faudrait vérifier la fréquence dans l'ensemble des poèmes de Mahon) pourrait laisser entendre que, contrairement à la critique qui insiste sur la séparation du poète de sa communauté, cette séparation ne va pas de soi.

(16) a West Side Story love scene on the sidewalk, / whoop of police sirens, car alarms / unanimous as in a California quake / while most lay dreaming in each other's arms. (77-138)

(16a) scène d'amour de West Side Story sur le trottoir, / hululements de sirènes, alarmes de voiture / unanimes comme pour un séisme californien, / quand tant de gens rêvent tendrement enlacés. (77a-139)

(17) It used to be, with characters like us, / they'd let us wander the roads in wind and rain / or lock us up and throw away the key - (85-146)

(17a) Il fut un temps où les gens comme nous, / on les laissait divaguer dans la pluie et le vent, / ou bien on les coffrait et on jetait la clé - (85a-146a)

Ces deux derniers exemples (16/16a) et (17/17a), marginaux au regard du choix de man et people, figurent ici en raison du nom d'humain général gens, l'équivalent de people, mais qui traduit ici le pronom most et le nom d'humain characters. En (16/16a) most et tant de gens occupent la fonction de sujet syntaxique et en (17/17a) characters like us / les gens comme nous est à la fois complément d'objet direct des verbes divaguer et coffrer (le pronom anaphorique us / les en est le relais) et le sujet logique de l'énoncé. Most renvoie à une grande quantité de personnes, characters à un type de personnes particulier, doté de qualités qui le distinguent des autres types. Ces qualités sont, dans cet extrait, celles qui caractérisent l'énonciateur (us/nous). La comparaison apparait cruciale dans l'interprétation du syntagme en vue de sa traduction car, en effet, sans l'identification marquée par like/comme, la traduction de characters par gens ne serait pas possible. La comparaison fonctionne comme une expansion de characters délimitant sa référence. Les gens sans l'expansion dénoterait un référent pluriel sans qualités particulières alors qu'ici les particularités et caractéristiques de us/nous donnent à l'ensemble visé une 
homogénéité qui n'est pas le simple fait de ses éléments +humains mais le résultat d'un ensemble de propriétés partagées, celles qui caractérisent l'énonciateur. Curieusement, gens ici se rapprochent d'individu mais individu au lieu de gens aurait gommé la valeur d' « ensemble non individualisé ». Ces aspects, dans la mesure où gens est réputé renvoyer à un ensemble à la fois homogène (comme ici) et hétérogène, mérite d'être signalés car ils semblent contredire ce que Schnedecker relève à propos de gens: "Par ailleurs, Schnedecker (2012) a démontré que cet ensemble est homogène du fait de ses éléments humains, mais en même temps est hétérogène en ce sens que ceux-ci semblent appartenir à des sous-catégories différentes ${ }^{21} »$.

L'ensemble des extraits où le nom d'humain people est présent et les deux occurrences sémantiquement apparentées ajoutées à cette liste mettent en lumière la valeur relationnelle de ce nom d'humain (valeur sans doute sémantiquement inscrite dans son caractère de nom collectif), l'un des plus indéterminés qui soient. Comme précédemment, le co-texte immédiat et le contexte plus large du poème influent sur la façon dont chaque occurrence réfère et il apparait que le choix de traduction met en lumière ce qui pourrait constituer l'une des valeurs secondaires de l'item lexical.

Cette étude empirique d'énoncés extraits d'un corpus contraint (et limitant, du fait de sa nature, les manipulations), nous a permis de confronter des occurrences de man et people à une diversité de traductions proposées par un seul traducteur. La confrontation a mis en lumière des faits d'ordre général et particulier. Il est clair en effet qu'un choix de traduction juste éclaire l'original et la saisie des valeurs référentielles de noms aussi généraux que ceux étudiés ici en faisant ressortir certains traits sous-jacents aux valeurs centrales. Autrement dit, chaque occurrence, chaque énoncé portent une part d'implicite que le choix de traduction guidé par une interprétation peut révéler. Cependant, la traduction ne peut être juste que si tous les paramètres de l'original sont pris en compte et nous avons pu mesurer l'importance des déterminants et des expansions adjectivales ou propositionnelles qui délimitent les occurrences, les noms expansés s'avérant les plus nombreux.

S'agissant du sémantisme des noms d'humains, man et people, la confrontation nous a amenée à explorer, prudemment, la possibilité théorique d'appliquer la théorie des modalités extrinsèques et intrinsèques de Gosselin. Cette première tentative tâtonnante appelle d'autres investigations car elle déplace l'application stricte de cette approche qui vise à une classification motivée des noms d'humains dans leur ensemble, vers une utilisation visant, elle, à expliquer et justifier des choix de traduction motivés non seulement par des contraintes sémantiques ou syntaxiques, mais dans le cas de ce corpus, par des considérations prosodiques évidentes, ces choix faisant apparaittre, par ricochet, les problèmes que la compréhension de l'humain à travers sa représentation linguistique pose.

41 Enfin, ce travail limité à quelques noms d'humains ne permet pas encore de vérifier l'hypothèse interprétative de départ, mais ce volet herméneutique déborde le seul périmètre de la linguistique et nécessite l'examen systématique de toutes les occurrences de noms d'humains rapportées à leur opposition éventuelle aux noms désignant des objets. 


\section{BIBLIOGRAPHIE}

CAPPEAU, P. \& SCHNEDECKER, C. (2014) : « Gens, personne(s), individu(s) : Trois saisies de l'humain ». In F. Neveu et al. (éds.) : Actes du 4ème Congrès Mondial de Linguistique Française, 19 - 23 juillet 2014, Berlin.

CHALKER, S., 1984, Current English Grammar, Londres/Basingstoke, MacMillan Publishers.

CULIOLI, A., 1984, « En guise d'introduction », La Langue au ras du texte, Lille, Presses Universitaires de Lille.

CUlioli, A., 1990, « La linguistique : de l'empirique au formel », Pour une linguistique de l'énonciation. Opérations et représentations, tome 1, Gap/Paris, Ophrys.

CULIOLI, A., \& NORMAND, C., 2005, Onze rencontres sur le langage et les langues, Paris, Ophrys.

DEPRAETERE, I., LANGFORD, C., 2012, Advanced English Grammar. A Linguistic Approach, Londres/New York, Continuum.

GOSSELIN, L., 2010, Les modalités en français, Amsterdam/New York, Rodopi.

GOSSELIN, L., 2015, Classes et classificateurs. Les modalités intrinsèques aux NH, Document interne au groupe de recherche NHUMA (non publié).

HUdDLESTON, R., \& PULLUM, G.K., 2012 [2002], The Cambridge Grammar of the English Language, Cambridge, Cambridge University Press.

MAHON, D., 2013, La Mer hivernale et autres poèmes, traduit de l'anglais (Irlande) et préfacé par J. Chuto, édition bilingue, Le Chambon-sur-Lignon, Cheyne éditeur.

NAUGRETTE-FOURNIER, M., 2015, Pour une nouvelle histoire des objets : réévaluation, classement et recyclage dans l'œuvre poétique de Derek Mahon, thèse de doctorat, non publiée.

QUIRK, R., GREEBAUM, S., LEECH, G., SVARTIK, J., 1972, A Grammar of Contemporary English, Harlow, Longman.

ROQUE AMARAL, E. T. \& MIHATSCH, W. 2016 : «Le nom français personne en comparaison avec le portugais brésilien pessoa et l'allemand Person : des noms en voie de pronominalisation? " : Actes du $5^{\mathrm{e}}$ Congrès Mondial de Linguistique Française, SHS Web of Conferences, Volume 27.

SCHNEDECKER, C. \& MIHATSCH, W., éds, 2015, Les Noms d'humains, une catégorie à part ?, Franz Steiner Verlag.

\section{NOTES}

1. Cette recherche collective rassemble des linguistes sémanticiens, morphologues, syntacticiens et informaticiens des universités de Strasbourg, Lorraine, Arras, Toulouse, Valenciennes, Poitiers et Rouen, de Tübingen, Chypre et Nagoya. Le projet s'intitule NHUMA.

2. On notera cependant une étude de Wiltrud Mihatsch (Groupe NHUMA), publiée en collaboration avec Eduardo Amaral, portant sur le nom français personne comparée avec le portugais brésilien pessoa et l'allemand Person. Voir bibliographie en fin d'article. 
3. Cette présente étude s'inscrit dans le cadre du projet cité mais aussi dans la continuité d'une recherche engagée dès notre thèse (2000) consacrée à l'étude du concept métalinguistique de «notion » (Théorie des Opérations Prédicatives et Énonciatives, d'Antoine Culioli) mis à l'épreuve d'un corpus poétique incluant notamment la poésie publiée de Derek Mahon à cette époque-là.

4. Une thèse de doctorat sur ce sujet a été soutenue le 7 décembre 2015 à l'Université Sorbonne Nouvelle: Pour une nouvelle histoire des objets: réévaluation, classement et recyclage dans l'œuvre poétique de Derek Mahon. Son auteur, Marion Naugrette-Fournier, se concentre sur le rapport du poète aux objets, délaissant la part «humaine» de cette poésie (et de ce rapport): «Paradoxalement, ce qui est lié à l'humain ne semble pas susciter d'émotion particulière chez Mahon, du moins en apparence, alors que les objets relevant du non-humain, voire même du posthumain, semblent toucher une corde sensible chez un poète qu'on a souvent décrit comme froid ou inaccessible. » (p. 20-21, thèse non publiée).

5. La principale difficulté étant l'exploration artisanale d'un relevé manuel en raison de la marge d'erreurs inhérente à ce mode d'observation ; inversement, la connaissance de l'ensemble permet de reconnaître les réseaux de signification internes à l'œuvre dans lesquels telle ou telle dénomination entre.

6. L'expression est empruntée à Culioli qui l'utilise à plusieurs reprises. Voir notamment (1990 : 35, 2005 : 146). Tous les corpus, quel que soit leur mode de collecte, sont, en réalité, contraints, notamment par la nature (langue écrite, langue parlée), le genre des textes collectés, les investigations qu'on y conduit et l'outil utilisé. Cependant, cette expression, qui différencie deux types de corpus, souligne que les données y sont traitées différemment. Dans ces textes écrits, les manipulations des énoncés, quand elles sont possibles, sont orientées vers leur interprétation tributaire de l'intégralité de leur contexte.

7. Cité par Schnedecker (2015) p. 27.

8. The New Oxford Dictionary of English (1998).

9. The Oxford Advanced Learner's Dictionary (2005).

10. Dictionary of English Language and Culture (1993 [1992]).

11. Wordnet : http://wordnetweb.princeton.edu/perl/webwn (consulté le 25.01.2017).

12. Les variations typographiques de police internes aux citations reproduisent celles des dictionnaires consultés.

13. Ces chiffres renvoient pour le premier à l'ordre de la collecte et pour le second à la page du recueil. Figurent en italique les occurrences de noms d'humains qui, s'ils ne font pas l'objet d'une analyse, permettent de situer l'occurrence générale examinée.

14. Nous faisons référence ici à un document de Laurent Gosselin intitulé «Contribution à la recherche sur les $\mathrm{NH}$ », daté du 16.01.2015 et communiqué au groupe de recherche impliqué dans le projet NHUMA. Ce travail applique aux noms d'humains la théorie des modalités intrinsèques développées dans Gosselin 2010.

15. L'anaphore est, semble-t-il, partielle : celui renvoie à une occurrence d'homme correspondant au prototype mais aussi, en tant qu'antécédent du pronom relatif, à cet individu que, précisément, la relative décrit.

16. Il s'agit en réalité d'une évocation de l'Irlande du Nord au moment des « Troubles ».

17. Voir notes 8 à 11 supra.

18. Le premier est extrait d'un poème dans lequel l'énonciateur-poète exprime violemment son rejet de la communauté protestante, puritaine et hypocrite dont il est membre. Le second est extrait d'un poème inspiré par une description des Rois du Feu du Cambodge dans The Golden Bough (Le Rameau d'Or) de James G. Frazer, description qui se superpose aux fantasmes du poète.

19. Le Nouveau Petit Robert de la Langue Française, $1993: 1653$.

20. Il n'est pas certain cependant que le réagencement syntaxique opéré dans l'extrait (11/11a) permette de saisir la concordance et l'identification entre Your people et credulous people.

21. Cité dans Cappeau \& Schnedecker, 2014, p. 11. 


\section{RÉSUMÉS}

Cet article vise à introduire dans l'étude des noms d'humains les paramètres du genre textuel et de la contrastivité à partir de l'étude d'un «corpus contraint» limité à un recueil bilingue de poèmes de Derek Mahon, poète irlandais, traduit en français par Jacques Chuto. Ce choix est motivé, sur le plan de l'interprétation de cette poésie, par le constat d'une asymétrie entre les occurrences de noms d'humains et celles de noms d'objets et, sur le plan linguistique par la nouveauté que représente l'étude contrastive des noms d'humains et de leur traduction. Ce travail de mise au jour des valeurs référentielles des noms d'humains enrichira les études en cours en y intégrant une approche contrastive, ce qui n'a pas encore été fait de manière extensive, et en ajoutant aux études des noms de l'anglais celle de la sous-catégorie des noms d'humains.

The aim of this paper is to contribute a contrastive analysis of a neglected class of nouns specifically denoting human beings. The material under scrutiny is a restricted number of occurrences in a selection of poems by the well-known Irish poet and translator Derek Mahon and its translation into French by Jacques Chuto. This particular choice is prompted by the fact that Mahon's poetry is a poetry of objects and, as a consequence, the mention of humans is deemed rather scarce. The aim of the present study however is not to verify this hermeneutic hypothesis which requires an in-depth exploration of the whole œuvre but, from a linguistic and semantic perspective, to bring to light the invariant and variant meanings of the nouns denoting humans which are present in the poems by contrasting them to their translation.

\section{INDEX}

Mots-clés : noms généraux d'humains, contraste anglais-français, corpus littéraire, traduction

\section{AUTEUR}

\section{MARYVONNE BOISSEAU}

Université de Strasbourg, UR LiLPa - EA 1339 - ER « Fonctionnements discursifs et Traduction » 